\title{
MRA evaluation of cerebrovascular stenosis and its association with acute stroke in Indian patients
}

\author{
Parida K. ${ }^{1}$, Biswal D. ${ }^{2}$ \\ ${ }^{1}$ Dr. Kalyani Parida, Associate Professor, Radiology, SCB Medical College \& Hospital, Cuttack, Odisha, India, \\ ${ }^{2}$ Dr. Dvijottam Biswal, 3rd Year Student, IMS \& SUM Hospital, Bhubaneswar, Odisha, India.
}

Address for Correspondence: Dr. Kalyani Parida, Email: drkalyaniparida@gmail.com

\begin{abstract}
Introduction: The detection of intracranial atherosclerotic disease has been elusive, possibly because of the dearth of non-invasive methods for diagnosing the condition. Material and Methods: In this study, we used time-of-flight MRA (TOF-MRA) to identify the presence, nature, and number of stenosis in the intra and extracranial arteries and anterior and posterior circulation systems in 60 Indian patients. Results: It was found that there is a strong association between the presence of stenosis and hyperlipidemia and hypertension. Intracranial stenosis was more prevalent in the $>60$ years age group, and it maximally affected the M1 segment of the middle cerebral artery. Comparatively, significant stenosis and total occlusion were predominant in the intracranial system; single stenosis was associated with the intracranial arteries, whereas multiple stenoses were observed in the extracranial system. Conclusion: Hemiparesis was the most common symptom of the patients. Thus, TOF-MRA can be used successfully to evaluate the occurrence, prognosis, and treatment options for cerebrovascular stenotic diseases.
\end{abstract}

Keywords: Intracranial stenosis, intracranial atherosclerotic disease, time-of-flight MRA, cerebrovascular stenotic diseases, stenosis

\section{Introduction}

Strokes are caused when the blood flow to the brain is interrupted, thus, resulting in cell death. The World Health Organization (WHO) defined stroke as a "neurological deficit of cerebrovascular cause that persists beyond 24 hours or is interrupted by death within 24 hours" [1].

Stroke can be ischemic or hemorrhagic. Ischemic stroke arises from thrombosis, embolism [2], systemic hypoperfusion [3] or cerebral venous sinus thrombosis [4]. Symptoms include difficulty in speaking, paralysis on one or both sides of the body, ambulatory problems, vision problems, and headache. In thrombotic stroke, a blood clot is formed around the atherosclerotic plaques, resulting in stenosis. An impending case of thrombotic stroke can be detected using various imaging techniques after assessing the patient's medical history. Thrombotic stroke can occur due

Manuscript received: $19^{\text {th }}$ June 2017

Reviewed: $30^{\text {th }}$ June 2017

Author Corrected: $9^{\text {th }}$ July 2017

Accepted for Publication: $17^{\text {th }}$ July 2017 to stenosis in the large vessels like the common carotid and internal carotid arteries (ICA), Circle of Willis, and the vertebral artery. It can also occur in smaller vessels such as middle cerebral artery (MCA), branches of the Circle of Willis, basilar arteries, and arteries arising from the distal vertebral.

Stroke is diagnosed using various imaging methods including computed tomography (CT) scan, magnetic angiography (MRA), magnetic resonance imaging (MRI), Doppler/duplex ultrasound, and arteriography. For the ischemic stroke diagnosis, CT scan has $16 \%$ sensitivity and $96 \%$ specificity. In contrast, MRI has $83 \%$ sensitivity and $98 \%$ specificity [5]. In addition, CT involves the use of X-rays, which may not be suitable for certain patients. Therefore, non-invasive, non-irradiationbased, and highly sensitive imaging methods are preferred for the diagnosis of cerebrovascular conditions. Currently, MRI, MRA, and Doppler/duplex ultrasonography are used to assess 
the presence, location, and extent of stenosis in symptomatic and asymptomatic patients with stroke, and this might assist in deciding the mode of treatment [6,7]. Intracranial stenosis (ICS) is predominant among Indian patients and is in contrast to that observed in Caucasian and Oriental patients [8, 9]. However, detection of intracranial atherosclerotic disease has been elusive, possibly because of the dearth of non-invasive methods for diagnosing the condition. MRI, MRA, and duplex ultrasonography have been used to detect intra and extracranial stenosis in the Indian population. Here, we used time-of-flight MRA (TOF-MRA) and MRI to detect and analyze stenosis and infarction in Indian patients enrolled at the SCB Medical College, Cuttack, India. We obtained valuable information regarding the age distribution of cases, risk factors predisposing towards stenosis and ischemic stroke, and the arterial locations, number, and significance of the stenosis.

\section{Material and Methods}

Data source: This study was conducted in the Department of Radiodiagnosis, SCB Medical College, Cuttack, Odisha, India between May 2013 and July 2015. Data were collected from 60 patients who were clinically diagnosed to have had stroke. All patients underwent MRI and MRA.

Informed consent was obtained from each patient before the study. The work presented in this study conforms to the guidelines of the institutional ethics committee of SCB Medical College, Cuttack.

Study Design: Prospective observational study

Inclusion Criteria: All subjects exhibited central nervous system signs of stroke. Males and females of all age groups who showed neurological symptoms of acute stroke and were subjected to diagnostic imaging methods within 72 hours of exhibiting the symptoms were included in the study.

Exclusion criteria: Patients who had hemorrhagic stroke, had suffered from an attack 30 days prior to the commencement of the study, had undergone any intracranial or interspinal surgery or head trauma in past 3 months, or had any contraindications to MRI were excluded from the study.
Pre-procedural assessment: The medical history of all the patients were assessed and recorded. In addition, the patients were clinically examined before any imaging procedure. Following are the parameters that were assessed:

1. Hypertension: Systolic blood pressure $>140 \mathrm{~mm}$ $\mathrm{Hg}$ and diastolic pressure $>90 \mathrm{~mm} \mathrm{Hg}$ in two independent sessions of blood pressure monitoring that required antihypertensive medication.

2. DM: Fasting blood glucose level $>125 \mathrm{mg} / \mathrm{dL}$ in two separate tests or requirement of antidiabetic medication before admission.

3. Hyperlipidemia: History of abnormally high lipids that required dietary or pharmacological intervention.

Procedure: MRI was performed using a GE Sigma HDX MR machine that had a 1.5 tesla field strength magnet. Multiplanar scout sections were obtained for planning the sequences. MRI was performed from the vertex of the brain to the foramen magnum, including the base of the skull, using axial, coronal, and sagittal sections.

MRA was performed using the 3-D time of flight technique and a standard head coil. Two different sets of MRAs were performed at the cervical carotid arteries and the Circle of Willis. 3-D images were acquired in the axial planes using echo technique with spoiling and flow compensation.

All intra- and extracranial findings were grouped into symptomatic or contralateral findings based on the clinical history. Stenosis measurements were computed directly on the maximum intensity projection views of MRA.

The percentage of stenosis was computed by measuring the diameter of the residual lumen at the site of maximum stenosis in each segment of the artery and then dividing the difference (with respect to the original diameter) by the original diameter. Those vessels that had a signal void on MRA and had evidence of regained flow distal to the proximal part were considered.

Statistical Analysis: All the data are expressed as percentages of the total. Microsoft Excel was used to plot the data obtained from MRI and MRA. 


\section{Results}

Age distribution of cases: Sixty patients who were evaluated to have suffered from stroke using MRA were included in this study. Fifty six patients $(93.34 \%$, median age 59 years) were above 50 years of age. Only 4 patients $(6.67 \%)$ were $<50$ years. Twenty six patients $(43.34 \%)$ were in the age group of $50-60$ years.

Distribution of cases according to stenotic site: Table 1 presents the distribution of cases according to stenotic site and sex. The presence of stenosis in the internal and external carotid arteries in $<60$ year age group and $>$ 60 year age group were assessed using TOF-MRA. External carotid stenosis (ECS) was more common (approximately double) in the $>60$ years age group than in the $<60$ years group (Table 1). In contrast, ICS was slightly more prevalent in the $<60$ years age group (Figure 1). Both groups had similar probabilities of having either no stenosis or both ICS and ECS.

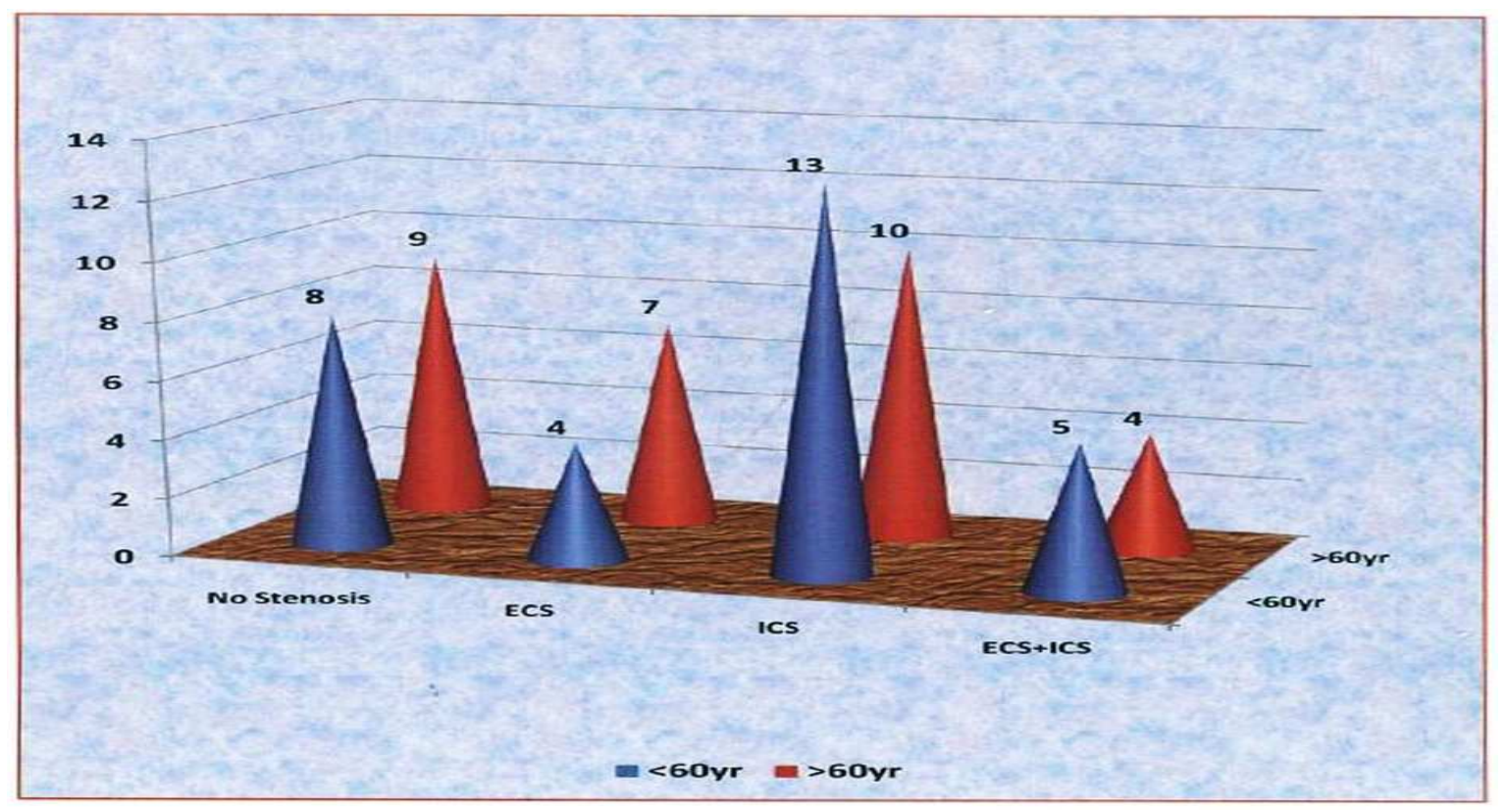

Figure 1: Extracranial and intracranial stenosis (ECS and ICS, respectively) were assessed using TOF-MRA in patients who were either $<60$ years or $>60$ years old $(\mathrm{N}=30$ per group). The resulting data was plotted as histograms using Microsoft Excel.

Table-1: Distribution of cases according to stenotic site.

\begin{tabular}{|c|c|c|c|c|}
\hline \multirow{2}{*}{ Site of stenotic lesion } & \multicolumn{2}{|c|}{ Age } & \multicolumn{2}{c|}{ Sex } \\
\cline { 2 - 5 } & $<\mathbf{6 0}$ years & $>\mathbf{6 0}$ years & Male & Female \\
\hline Normal (no stenosis): 17 & 8 & 9 & 11 & 6 \\
\hline Isolated ECS: 11 & 4 & 7 & 9 & 2 \\
\hline Isolated ICS: 23 & 13 & 10 & 14 & 9 \\
\hline Both ICS and ECS: 9 & 5 & 4 & 7 & 2 \\
\hline
\end{tabular}

Distribution of cases according to sex: Table 1 also presents the distribution of cases according to sex. We analyzed 41 males and 19 females for the occurrence of ECS, ICS, ECS + ICS or no stenosis. Males dominated all these categories compared to females. ICS appeared to be more predominant in both males and females.

Frequency of different stroke symptoms: We evaluated the frequency of reporting hemiparesis, monoparesis, paraparesis, headache, cerebellar symptoms, vision loss, syncope, and dizziness in the patients. Hemiparesis was the most reported symptom among all patients (26.7\%), followed by monoparesis $(21.7 \%)$ (Table 2). 
Table-2: Frequency of different symptoms \& signs in patients with cerebral infarction.

\begin{tabular}{|c|c|c|}
\hline Symptoms \& sign & No of patients $(\mathbf{6 0})$ & $\mathbf{\%}$ \\
\hline Hemiparesis & 18 & 30 \\
Right side & 16 & 26.7 \\
Left side & 13 & 21.7 \\
\hline Monoparesis & 1 & 16.7 \\
\hline Paraparesis & 5 & 1.65 \\
\hline Headache & 3 & 8.3 \\
\hline Cerebellar symptoms & 3 & 55 \\
\hline Vision loss & 1 & 1.65 \\
\hline Syncope, dizziness & & \\
\hline
\end{tabular}

Association of hypertension with stenosis: We correlated the blood pressure records of 60 patients with the occurrence of stenosis and grouped them into "stenosis present" and "stenosis absent" groups. Out of 47 hypertension patients, $37(78.7 \%)$ were in the "stenosis present" group and only $10(21.3 \%)$ were in the "stenosis absent" group. On the other hand, for the 13 non-hypertension patients, there was almost equal distribution, with $6(46.1 \%)$ patients in the "stenosis present" group and $7(53.9 \%)$ patients in the "stenosis absent" group.

Association of diabetes mellitus (DM) with stenosis: To investigate the relationship of DM with stenosis, we categorized the patients into DM+ve and DM-ve groups of 30 patients each and correlated the presence of ECS, ICS, ECS + ICS or no stenosis in both groups. Out of them ICS is seen in $11(36.67 \%)$ cases, ECS in 5 cases and both in 4 cases and there is no stenosis in 9 cases. This suggests that ICS is more common in patients with diabetes than ECS (Table 3). However, larger number of patients may have to be evaluated to obtain significant results in this respect.

Table-3: Distribution of stenosis in DM patients

\begin{tabular}{|c|c|c|}
\hline \multirow{2}{*}{ Site of stenotic lesions } & \multicolumn{2}{|c|}{ DM Patients } \\
\cline { 2 - 3 } & + ve & -ve \\
\hline Normal (No stenosis ): 17 & 10 & 7 \\
\hline ECS isolated: 11 & 5 & 6 \\
\hline ICS isolated: 23 & 11 & 5 \\
\hline Both ICS and ECS: 9 & 4 & 12 \\
\hline
\end{tabular}

Association of lipid profile with stenosis: We evaluated the association of low-density lipoprotein (LDL), high-density lipoprotein (HDL), and total cholesterol content with absence or presence of stenosis in the patients. Stenosis was significantly associated with low HDL (90.7\% vs. 23\%; stenotic vs. non-stenotic) and high LDL (93\% vs. 76.7\%; stenotic vs. non-stenotic) levels (Tables 4). In the group of patients with increased total cholesterol, $81.4 \%$ had stenosis and $88.2 \%$ did not have stenosis, which is not a statistically significant difference. Larger number of patients have to be evaluated to derive any conclusion on this aspect.

Table-4: HDL, LDL and total CHL level in stenosis and non-stenosis patients

\begin{tabular}{|c|c|c|c|c|c|c|c|}
\hline SI & \multirow{2}{*}{ Patients } & \multicolumn{2}{|c|}{ HDL $^{*}$} & \multicolumn{2}{c|}{ LDL** } & \multicolumn{2}{c|}{ Total CHL } \\
\cline { 3 - 7 } & Decreased & Normal & Increased & Normal & Increased & Normal \\
\hline 1 & $\begin{array}{c}\text { Stenosis present } \\
(43 \text { patients })\end{array}$ & $39(90.7 \%)$ & $4(9.3 \%)$ & $40(93 \%)$ & $3(7 \%)$ & $35(81.4 \%)$ & $8(18.6 \%)$ \\
\hline 2 & $\begin{array}{c}\text { Stenosis absent } \\
(17 \text { patients })\end{array}$ & $4(23.3 \%)$ & $13(76.7 \%)$ & $13(76.7 \%)$ & $4(23.3 \%)$ & $15(88.2 \%)$ & $2(11.8 \%)$ \\
\hline
\end{tabular}


Distribution of infarcts in different arterial territories: We evaluated the presence of stenosis in the anterior cerebral artery (ACA), posterior cerebral artery (PCA), middle cerebral artery (MCA), internal carotid artery (ICA), watershed infarcts, and ischemic lesions using MRI in 54 patients. MCA was the most severely affected artery $(61.1 \%$ ) (Figures $2 \mathrm{~A}$ ), which resulted in infarction of the right temporal lobe (Figures $2 \mathrm{~B}, \mathrm{C}$ ) and the fronto-parietal and right parietal lobes (Figures 2D, E), followed by the PCA, which affected the occipital lobe (15\%) (Figure 3, Table 5). MRA showed that M1 was the most common site of stenosis in MCA (78.3\%) (Figure 3D). M2 was stenosis site in $8.6 \%$ whereas $\mathrm{M} 1+\mathrm{M} 2$ was in $13.1 \%$.

Table-5: Distribution of Infarcts in different arterial territories according to MRI findings

\begin{tabular}{|c|c|c|}
\hline Arterial territory & Total (54) & \% \\
\hline ACA & 2 & 3.7 \\
\hline MCA & 33 & 14.8 \\
\hline PCA & 8 & 7.4 \\
\hline ACA+MCA & 4 & 5.6 \\
\hline Watershed infarcts & 3 & 7.4 \\
\hline Ischemic lesions & 4 & 61.6 \\
\hline
\end{tabular}

Distribution of cases according to MRA: MRA showed that among the 54 cases of stenosis plus infarcts, clinically significant stenosis (including cases of total occlusion 10 cases and significant stenosis 25 cases) occurred in $65 \%$ of the cases. Non significant stenosis occurred in 8 patients $(14.8 \%)$ and no stenosis in 11 patients $(20.37 \%)$.

Distribution and lateralization of extracranial carotid lesions: We analyzed the location of extracranial carotid lesions in 27 patients. The carotid bifurcation was the most common site of the lesion in (77\%) patients compared to the carotid siphon in $3(11 \%)$, and the proximal cervical carotid in $3(11 \%)$ patients. We also analyzed the occurrence of lesions on the left, right, and both sides (bilateral). Results showed that ECS stenotic lesions were predominantly distributed in 8 (29.6\%) patients on right side, 7 (26\%) on left side, and $6(22 \%)$ patients on both sides; i.e., $15(55.6 \%)$ for unilateral and $22 \%$ for bilateral).

Distribution of isolated intracranial and extracranial stenosis: We evaluated 43 cases of isolated intra- and extracranial stenosis using MRA. Intracranial stenosis in $23(53.5 \%)$ patients was more prevalent than extracranial stenosis in $11(25.5 \%)$ patients. Both intracranial and extracranial was in $9(21 \%)$ patients.

Distribution of significant stenotic lesions: We analyzed the distribution of significant stenotic lesions in MCA, PCA, ICA, ACA, ACA+MCA, and the vertebral artery. MCA had the maximum number of significant stenotic lesions (22\%), followed by ICA (11\%) (Table 6)

Table-6: Distribution of significant stenosis lesions

\begin{tabular}{|c|c|}
\hline Arterial territory & Total (44) \\
\hline MCA & 22 \\
\hline ICA & 11 \\
\hline PCA & 5 \\
\hline ACA & 2 \\
\hline MCA+ACA & 3 \\
\hline Vertebral & 1 \\
\hline
\end{tabular}

Distribution of significant stenotic lesions in the anterior and posterior circulation: We evaluated the occurrence of stenotic lesions in the anterior (MCA, ICA, ACA) and posterior circulatory loops (vertebral artery and PCA) and observed that the lesions were more common in $38(86 \%)$ in the anterior circulatory system (Figures 2) than in the posterior system in $6(14 \%)$ cases 
Distribution of cases according to number of stenosis: Among the 43 cases analyzed, we observed that multiple stenotic lesions were predominant in the extracranial system in $12(67 \%)$ patients (Figure 4) than in the intracranial system in $6(33 \%)$ patients, which had more instances of single lesions $(80 \%)$.

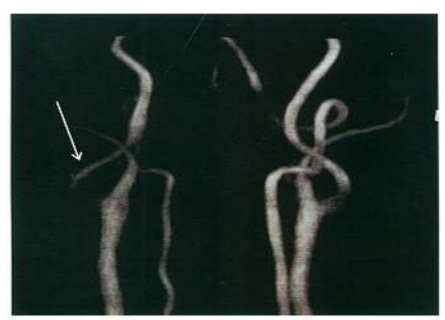

(A)

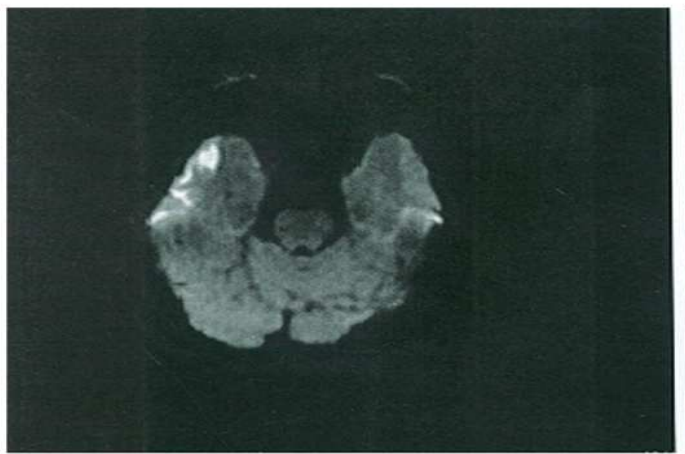

(D)

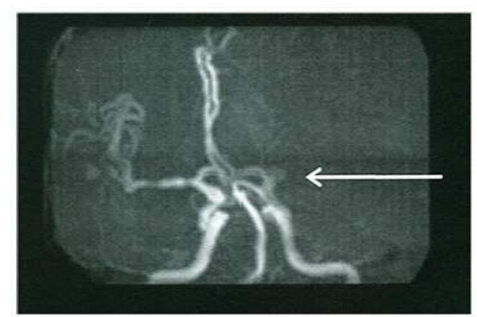

(B)

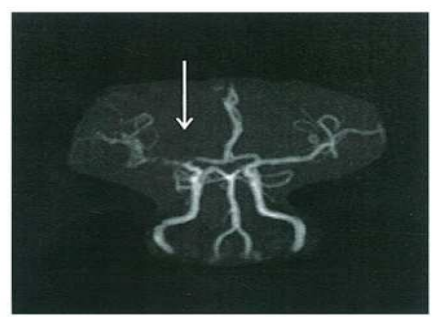

(C)

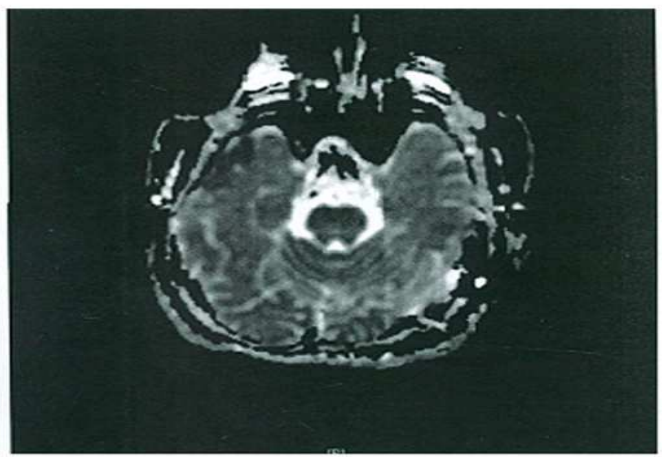

(E)

Figure 2: MRA showing luminal narrowing at the right cervical internal carotid artery at origin. (A) The narrowing, as seen by the reduction in signal intensity, is indicated by an arrow. (B)The middle cerebral artery (MCA) is the most severely affected arterial region inside the cranium. MRA showing stenosis in the MCA. MRA showing total occlusion in left MCA (indicated by arrow). (C) MRA showing luminal narrowing with reduced signal intensity in right MCA (indicated by arrow). (D) The areas of the brain supplied by the middle cerebral artery (MCA) are the most severely affected regions. MRI showing right temporal lobe infarction due to stenosis in the MCA. DWI showing diffusion restriction. (E) ADC image showing hypointensity.

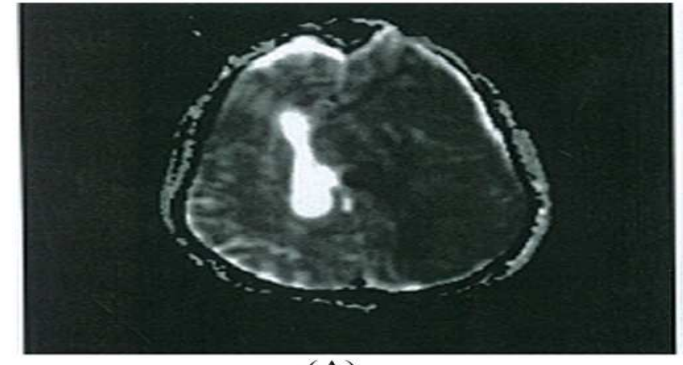

(A)

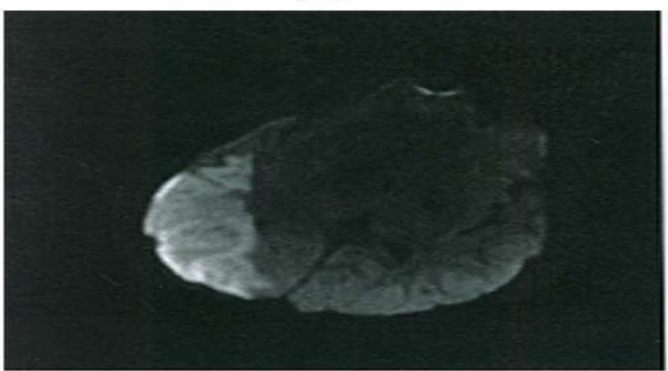

(C)

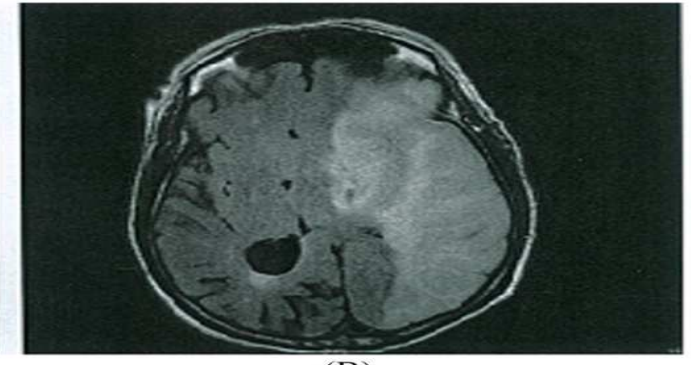

(B)

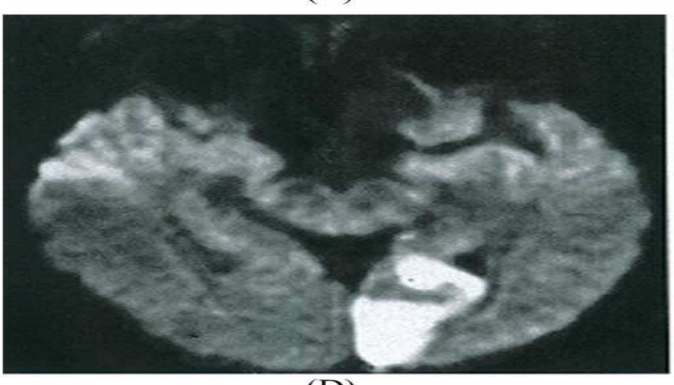

(D)

Figure 3: MRI showing infarction in right parietal and fronto-parietal lobes due to MCA stenosis. (A) ADC image showing hypointensity. (B) FLAIR image showing hyperintensity. (C) DWI showing diffusion restriction in right parietal lobe. (D) MRI showing infarction in the left occipital lobe due to posterior cerebral artery (PCA) stenosis with DWI showing diffusion restriction. 


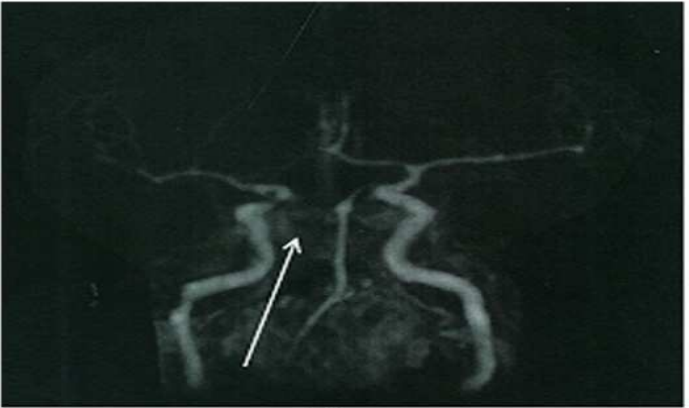

(A)

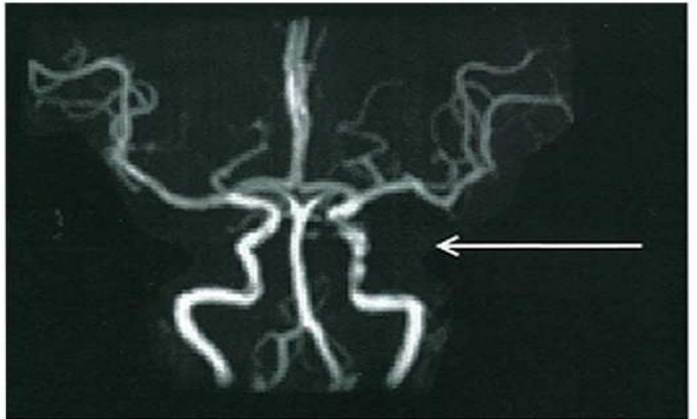

(C)

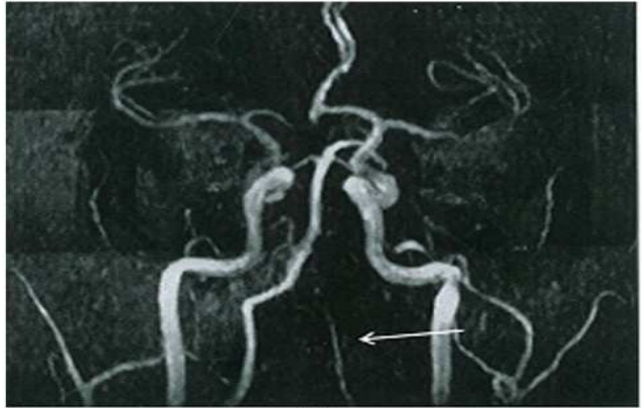

(B)

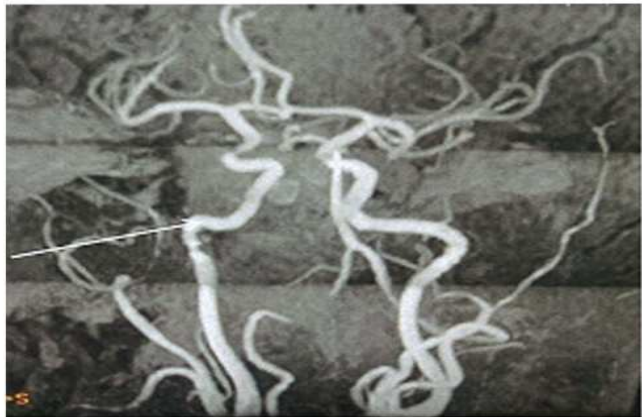

(D)

Figure 4: Stenosis in the posterior circulation is less frequent than in the anterior circulation. (A) MRA showing signal void in right PCA. (B) MRA showing luminal narrowing of the left vertebral artery (arrow). (C) MRA showing multiple left ICA stenosis. (D) MRA showing multiple right ICA stenosis.

\section{Discussion}

In this study, we examined the location and distribution of vascular lesions in Indian patients suffering from cerebrovascular steno-occlusive diseases using MRI and MRA. MRA records blood flow as normal or obstructed; obstruction can be due to clinically non-significant stenosis $(<50 \%$ stenosis), clinically significant stenosis ( $>50 \%$ stenosis), and total occlusive stenosis [6]. MRA can detect stenosis both in intracranial and extracranial vessels and in the anterior and posterior circulations.

Age and occurrence of stenosis: We investigated various aspects of stenosis in 60 patients who had undergone stroke and fit the inclusion criteria of our study. We found that majority of the patients who had stroke were 50-60 years or more than 60 years-old (median age 59 years), which is similar to the observations of Suh et al. [10] (median age of 268 patients was 56 years).

Further, we observed that ICS had an earlier onset as it was more common in the $<60$ years age group, whereas ECS occurred in the $>60$ years group. Two diagnostic studies performed on different subsets of the Indian population also showed higher representation of ICS-MRA anomalies in individuals affected with stroke, which corroborates our findings [8,9]. In addition, we also observed that stenosis predominantly occurred in males than in females.

Risk factors for carotid stenosis: Hypertension is a major risk factor for cerebrovascular diseases [6]. DM is another risk factor for cerebrovascular diseases [8, 9]. Transcranial Doppler examination was used to identify stenosis in MCAs in over onefifth of the Chinese type 2 diabetic subjects who had no symptoms of cerebrovascular disease. Gillard et al. [7] reported $43 \%$ cases where history of diabetes coincided with cerebral ischemia using MRA. In a large database survey, Newman et al. [11] showed that the probability of developing carotid artery stenosis increased for participants with diabetes alone. Surprisingly, we did not observe any difference between the associations of DM with stenosis in our study. Analysis of larger number of participants is required to draw definite conclusions in this regard.

Arterial locations of stenosis: MCA was the predominant location of stenosis $(61.1 \%)$, followed by PCA $(14.8 \%)$ in our study, which correlates well with previous observations [9, 12]. In MCA, 
M1 was the most common site $(78.3 \%)$, which is similar to that reported earlier [10]. Bilateral extracranial stenosis was common among our study participants, and the carotid bifurcation was the most common site of ECS $(77.7 \%)$. This agrees with the observations mentioned by Atlas et al., who found that atherosclerotic occlusive disease in the carotid siphon was only second to that in the carotid bifurcation [13].

Cases of isolated ICS were higher in our study $(53.5 \%)$. This is similar to the results of a largescale analysis, where among 1,955 patients, 77.6\% had atherosclerotic lesions in one or more extra- or intracranial arteries, and $39 \%$ of the patients showed isolated ICS [14].

Clinical types of stenosis: We observed that $43.3 \%$ of our patients had clinically non-significant stenosis $(0-49 \%$ stenosis $)$, whereas $40 \%$ had clinically significant stenosis (> 50\% stenosis) and $16.7 \%$ had total occlusion of vessels. The anterior circulation developed more significant stenosis (86\%) than the posterior circulation, which agrees with the results of Suh et al. [10], who reported $59 \%$ cases of significant stenosis in the anterior circulation and $41 \%$ in the posterior circulation.

Number and location of stenosis: We observed higher percentage of cases with single stenosis in the intracranial system than the extracranial system. Suh et al. [10] reported that among 56 patients with single severe stenosis, ICS was more frequent than ECS (66\% vs. $34 \%)$. In contrast, Zarei et al. [12] found multiple lesions to be abundant intracranially. These differences could arise because of differences in the number of subjects, ethnicities, and socio-physical factors.

\section{Conclusion}

Systematic surveys regarding the distribution of stroke-affected patients in various age groups and assessment of the role of risk factors in development of stenosis are limited in Indian medical research. Furthermore, MRA is rarely used as a tool for detecting asymptomatic cerebrovascular diseases or for determining the prognosis and treatment of such diseases. Considering the non-invasive nature of the technology, we used TOF-MRA for analyzing various aspects of cerebral stenosis in an Indian population and correlated the occurrence of stenosis with age, sex, hyperlipidemia, DM, and hypertension. We detected the most commonly affected arterial regions in intracranial stenosis and associated the number of stenosis with arterial location.

However, our study is not without limitations. We analyzed a small number of patients, which comprised mostly males. This might have biased the results in general, and therefore, inclusion of larger number of subjects would have increased the confidence level of the study.

Nonetheless, high-resolution MRA such as TOFMRA can detect vascular occlusive diseases, which would enable early intervention, design of appropriate treatment strategies, and reduce the number of patients for the expensive conventional angiography and thrombolytic treatment.

\section{Abbreviations}

WHO: World Health Organization, DM: Diabetes Mellitus, ICA: Internal Carotid Arteries, MCA: Middle Cerebral Artery, MRA: Magnetic Angiography, DWI: Diffusion-Weighted MRI, ICS: Intracranial Stenosis, TOF-MRA: Time-ofFlight MRA, ECS: External Carotid Stenosis, LDL: Low-Density Lipoprotein, HDL: HighDensity Lipoprotein, ACA: Anterior Cerebral Artery, PCA: Posterior Cerebral Artery, ACAS: Asymptomatic Carotid Artery Stenosis, ICA: Internal Carotid Artery.

Funding: Nil, Conflict of interest: None

Permission of IRB: Not required

\section{References}

1. World Health Organization. Cerebrovascular Disorders (Offset Publications). Geneva: World Health Organization; 1978.

2. Donnan GA, Fisher M, Macleod M, Davis SM. Stroke. Lancet. 2008 May 10;371(9624):1612-23. doi: 10.1016/S0140-6736(08)60694-7.

3. Shuaib A, Hachinski VC. Mechanisms and management of stroke in the elderly. CMAJ. 1991 Sep 1;145(5):433-43.

4. Stam J. Thrombosis of the cerebral veins and sinuses. N Engl J Med. 2005 Apr 28;352(17): 1791-8. 
5.Chalela JA, Kidwell CS, Nentwich LM, et al. Magnetic resonance imaging and computed tomography in emergency assessment of patients with suspected acute stroke: a prospective comparison. Lancet. 2007;369(9558):293-298.

6. Liu HM, Tu YK, Yip PK, Su CT. Evaluation of intracranial and extracranial carotid steno-occlusive diseases in Taiwan Chinese patients with MR angiography: preliminary experience. Stroke. 1996 Apr;27(4):650-3.

7. Gillard JH, Oliverio PJ, Barker PB, Oppenheimer SM, Bryan RN. MR angiography in acute cerebral ischemia of the anterior circulation: a preliminary report. AJNR Am J Neuroradiol. $1997 \mathrm{Feb} ; 18(2): 343-50$.

8. Kumar G, Kalita J, Kumar B, Bansal V, Jain SK, Misra U. Magnetic resonance angiography findings in patients with ischemic stroke from North India. J Stroke Cerebrovasc Dis. 2010;19(2):146-152.

9. Shrivastava A, Srivastava T, Saxena R. CT angiographic evaluation of pattern and distribution of stenosis and its association with risk factors among Indian ischemic stroke patients. Pol J Radiol. 2016;81:357-362.

10. Suh DC, Lee SH, Kim KR, et al. Pattern of atherosclerotic carotid stenosis in Korean patients with stroke: different involvement of intracranial versus extracranial vessels. AJNR Am J Neuroradiol. 2003; 24(2):239-244.

11. Newman JD, Rockman CB, Kosiborod M, Guo Y, Zhong H, Weintraub HS, Schwartzbard A, Adelman MA, Berger JS. Diabetes mellitus is a coronary heart disease risk equivalent for peripheral vascular disease. Am Heart J. 2017 Feb; 184:114-120. doi: 10.1016/j.ahj.2016.09.002. Epub 2016 Sep 18.

12. Zarei H, Ebrahimi H, Shafiee K, Yazdani M, Aghili K. Intracranial stenosis in patients with acute cerebrovascular accidents. ARYA Atheroscler. 2008;3(4):206-210.

13. Heiserman JE, Masaryk TJ, Aygun N. MR Angiography: Techniques and Clinical Applications. In: Atlas SW, editors. Magnetic Resonance Imaging of the Brain and Spine Volume Two. $4^{\text {th }}$ Edition. Philadelphia: Lippincott Williams \& Wilkins, a Wolters Kluwer Publication. 2009. p. 856.

14. Kim YD, Choi HY, Cho HJ, Cha MJ, Nam CM, Han SW, Nam HS, Heo JH. Increasing frequency and burden of cerebral artery atherosclerosis in Korean stroke patients. Yonsei Med J. 2010 May;51(3):318-25. doi: 10. 3349/ ymj.2010.51.3.318.

\section{How to cite this article?}

Parida K, Biswal D. MRA evaluation of cerebrovascular stenosis and its association with acute stroke in Indian patients. Int J Med Res Rev 2017;5(07):675-683. doi:10.17511/ijmrr. 2017.i07.05. 